Rev. Int. Contam. Ambie. 35 (1) 7-23, 2019

DOI: 10.20937/RICA.2019.35.01.01

\title{
CONOCIMIENTOS, CONDUCTAS Y SÍNTOMAS DE INTOXICACIÓN AGUDA POR PLAGUICIDAS ENTRE PRODUCTORES DE TRES SISTEMAS DE PRODUCCIÓN AGRÍCOLAS EN LOS ALTOS DE CHIAPAS, MÉXICO
}

\author{
Héctor Ulises BERNARDINO-HERNÁNDEZ ${ }^{1 *}$, Ramón MARIACA-MÉNDEZ ${ }^{2}$, \\ Austreberta NAZAR-BEUTELSPACHER ${ }^{2}$, José David ÁLVAREZ-SOLÍS ${ }^{2}$, Arturo TORRES-DOSAL ${ }^{2}$ \\ y Crispín HERRERA-PORTUGAL ${ }^{3}$
}

${ }^{1}$ Facultad de Ciencias Químicas, Universidad Autónoma Benito Juárez de Oaxaca, Av. Universidad s/n, Col. Cinco Señores, 68120 Oaxaca de Juárez, Oaxaca, México

${ }^{2}$ El Colegio de la Frontera Sur (ECOSUR). Carretera Panamericana y Periférico Sur S/N, Barrio de María Auxiliadora, C.P. 29290, San Cristóbal de Las Casas, Chiapas, México

${ }^{3}$ Facultad de Ciencias Químicas, Universidad Autónoma de Chiapas Carretera a Puerto Madero Km 1.5, C.P. 30580, Tapachula, Chiapas, México

*Autor para correspondencia; hbernardino@yahoo.com

(Recibido junio 2017; aceptado abril 2018)

Palabras clave: horticultura, floricultura, maíz, insecticidas, fungicidas, herbicidas

\section{RESUMEN}

Se realizó un estudio transversal y comparativo de tres sistemas de producción (maíz, flores y hortalizas) en la región de los Altos de Chiapas, México. Los objetivos fueron caracterizar los plaguicidas utilizados, describir los conocimientos y conductas de su manejo, e identificar los síntomas de intoxicación aguda por dichos insumos entre los productores agrícolas. Para recabar la información se aplicaron 523 encuestas dirigidas a los jefes de familia responsables del uso de dichos productos. La baja escolaridad se asoció de manera significativa con los escasos conocimientos sobre su peligrosidad, principalmente entre los productores de hortalizas. En los tres sistemas agrícolas, las conductas de manipulación son inapropiadas, independientemente de la escolaridad de los usuarios. Los floricultores y horticultores están expuestos a insecticidas extremadamente peligrosos (categoría toxicológica CT I) y altamente peligrosos (CT II), así como a fungicidas ligeramente peligrosos (CT IV). Los campesinos dedicados al cultivo de maíz se exponen con frecuencia a herbicidas CT II, III y IV e insecticidas CT I. Se identificó una diversidad de síntomas de intoxicación aguda entre los agricultores, probablemente asociada a la diversidad de plaguicidas utilizados. Es urgente diseñar e implementar estrategias para promover el uso seguro de estos insumos químicos y disminuir los riesgos a la salud en la población usuaria.

Key words: horticulture, floriculture, corn, insecticides, fungicides, herbicides

\begin{abstract}
A transversal and comparative study was conducted in three production systems (corn, flowers and vegetables) in the Highlands of Chiapas, Mexico. The objectives were to characterize the pesticides used, describe the knowledge and behavior of their management, as well as to identify the symptoms of acute intoxication by these inputs among
\end{abstract}


agricultural producers. To collect the information, 523 surveys were applied to heads of families responsible for the use of said products. Low educational level was associated significantly with little knowledge about its dangerousness, mainly among vegetable producers. In the three agricultural systems, the handling behavior is inappropriate regardless of the schooling of the users. Growers and horticulturists are mainly exposed to extremely hazardous insecticides (toxicological category TC I) and highly dangerous (TC II), as well slightly dangerous fungicides (TC IV). Peasants engaged in the cultivation of corn are frequently exposed to herbicides TC II, III and IV, and insecticides TC I. A variety of symptoms of acute poisoning among farmers was identified, probably associated with the diversity of pesticides used. It is urgent to design and implement strategies to promote safe use of chemical inputs and reduce the risks to health of the user population.

\section{INTRODUCCIÓN}

Los plaguicidas se han empleado durante muchos años en la agricultura para controlar plagas y evitar la pérdida de cultivos, así como alcanzar su máxima producción y calidad. Su potencial tóxico está relacionado con su capacidad para controlar y destruir plagas y enfermedades; sin embargo, esta característica los hace potencialmente dañinos para la salud y el ambiente (Plenge-Tellechea et al. 2007). Es cierto que estos productos han mantenido y mejorado la productividad agrícola; pero, a la vez, han contribuido al deterioro ecológico y de la salud de los seres vivos que forman parte del ecosistema, incluyendo la salud humana (Schoijet 2011).

Los países en desarrollo usan alrededor de un cuarto del total de plaguicidas utilizados en el planeta; sin embargo, presentan más del $50 \%$ de las intoxicaciones y el $99 \%$ de muertes atribuibles a la exposición a estos compuestos (Tinoco 2005). Cada año se presentan en todo el mundo entre 500 mil y un millón de intoxicaciones con dichos productos, de las cuales $70 \%$ se deben a exposición ocupacional en ambientes agrícolas (Eddleston et al. 2002).

México inició el registro de casos de intoxicación aguda por plaguicidas (IAP) en 1993, con un total de 1576 casos. Esta cifra se incrementó a 67711 casos en todo el país durante el periodo 1995-2012. Los estados que consistentemente presentaron las tasas más altas de incidencia de IAP a lo largo de ese periodo fueron Nayarit, Colima, Morelos y Jalisco. Otros estados presentaron una tendencia hacia el incremento de IAP al final de dicho periodo, entre ellos Baja California Sur, Chiapas, Guanajuato, Guerrero, Hidalgo, Oaxaca, Querétaro y Sinaloa. El registro se realiza a través del Sistema Único de Información para la Vigilancia Epidemiológica de la Secretaría de Salud, mediante el cual se captan los casos de intoxicación de manera genérica sin considerar el agente específico causante. Dadas las dificultades para el diagnóstico oportuno de las intoxicaciones, es probable que haya un subregistro de dichos padecimientos (Gutiérrez 2013).

Las crecientes ventas de los plaguicidas en las últimas décadas evidencian su continua y permanente utilización a lo largo de todo el territorio mexicano (Nivia 2000). De esta manera, se han incorporado lentamente hasta llegar a zonas rurales habitadas por campesinos indígenas con escasos recursos económicos, particularmente en el sureste mexicano, lo que ha provocado la transformación de un sistema agrícola tradicional, con nula incorporación de insumos externos y autosostenible de manera natural, a un sistema convencional dependiente de insumos químicos, con repercusiones negativas hacia los recursos naturales y la salud de la población (Ancury et al. 2001).

Tal es el caso del estado de Chiapas, México, donde gran parte de la población rural e indígena se dedica a la agricultura. En dicha entidad existen pocos datos sobre la epidemiología de las IAP, así como las condiciones de uso, manejo y exposición a plaguicidas. Los escasos estudios disponibles se han centrado en algunas localidades de la región fronteriza, como la Frailesca y el Soconusco (Tinoco et al. 1993, Tinoco 2005, Herrera-Portugal et al. 2005, Ríos-González 2006, Ventura 2007), pero hay escasa información sobre otras regiones. En particular la región de los Altos, por sus condiciones de producción agrícola, características socioeconómicas y reportes del uso intensivo de estos insumos (Álvarez-Solís y Anzuento-Martínez 2004, Santiago-Lastra y PeralesRivera 2007), pudiera albergar localidades de alto riesgo respecto de la exposición a plaguicidas. En este contexto, el objetivo del presente estudio fue caracterizar los plaguicidas utilizados, describir los conocimientos y conductas relacionados con su uso y manejo, y conocer los síntomas de IAP en tres sistemas de producción agrícola de comunidades rurales de los Altos de Chiapas, México. 


\section{MATERIALES Y MÉTODOS}

El estudio cuantitativo de tipo transversal y comparativo se realizó en sistemas de producción (SP) ubicados en comunidades rurales de tres municipios de la región Altos de Chiapas, que se caracterizan por el predominio de los cultivos de hortalizas (Chamula), flores (Zinacantán) y maíz (Amatenango del Valle) (Fig. 1). Dichos asentamientos humanos forman parte del grupo de municipios indígenas con población en situación de pobreza y de muy alta marginación en el estado de Chiapas (CONEVAL 2012). Mediante un censo se visitaron todas las viviendas (unidades domésticas, UD) de las comunidades en estudio. Se excluyeron aquellas UD que no se dedicaban a la agricultura, no utilizaban plaguicidas o no quisieron participar en el estudio. La muestra se formó con 523 UD (Chamula, 116; Zinacantán, 149, y Amatenango del Valle, 258).
Al jefe de familia (JF) se le aplicó una cédula general estructurada con preguntas cerradas y abiertas formada por dos apartados. En el primero se recolectó información sociodemográfica (edad, sexo, nivel de escolaridad, recepción de apoyos gubernamentales y su destino) y la descripción del SP (superficie sembrada por cultivo, tipos de cultivo, frecuencia de ciclos al año, presencia de plagas y uso de plaguicidas). El segundo apartado contenía preguntas con respuestas dicotómicas que recogió información relacionada con los conocimientos y conductas sobre el uso, manejo y almacenamiento de plaguicidas; aspectos vinculados con el aprendizaje y capacitación; hábitos de higiene; protección personal; almacenamiento y eliminación de envases vacíos, y presencia de síntomas de IAP durante o después de la fumigación. Dicho instrumento fue validado previamente en una comunidad con características similares a las del estudio. Las entrevistas fueron aplicadas por personal previamente

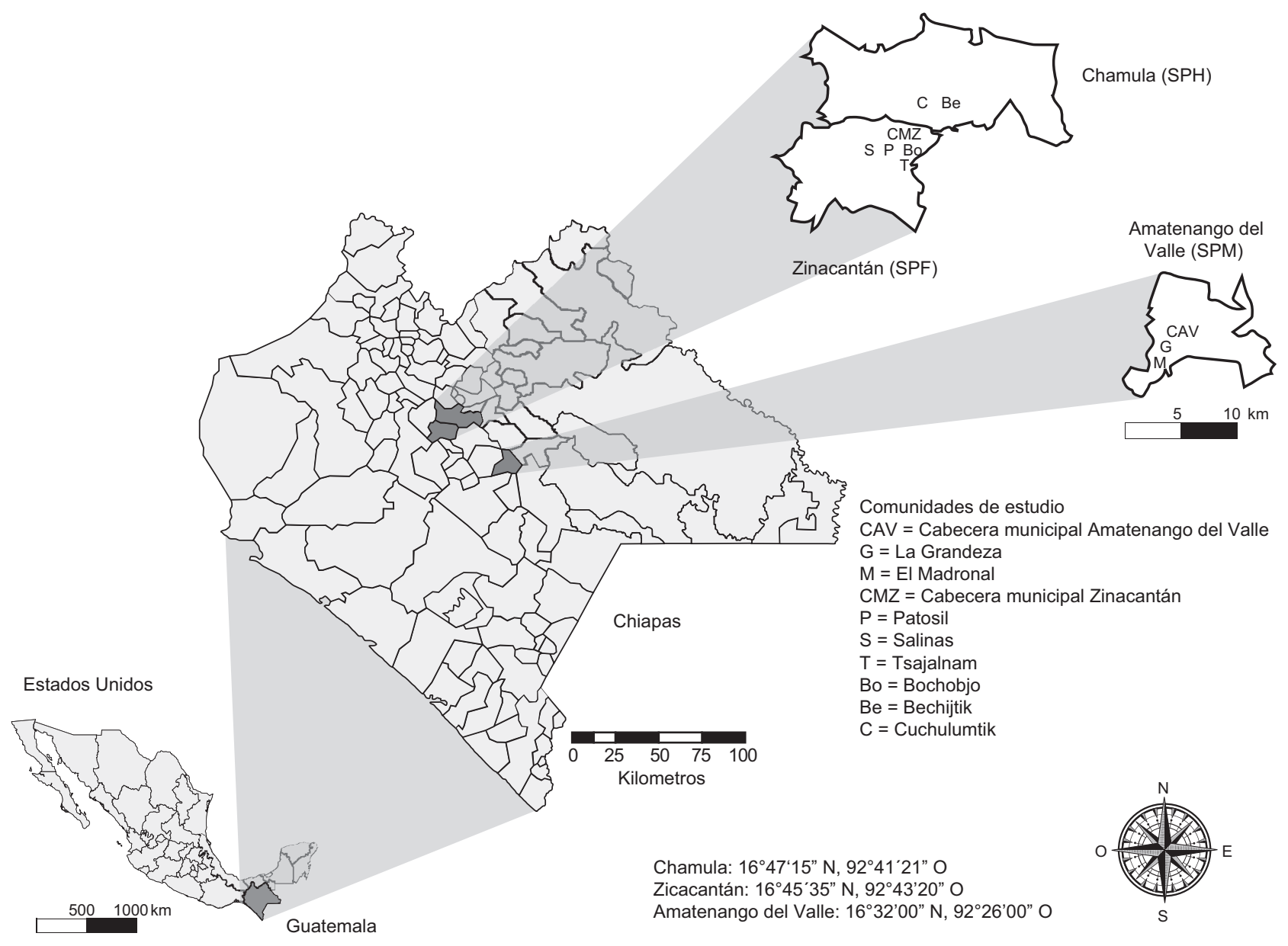

Fig. 1. Ubicación geográfica de los sistemas de producción estudiados. SPH: sistema de producción de hortalizas, SPF: sistema de producción de flores, SPM: sistema de producción de maíz 
capacitado y hablante de la lengua indígena de cada comunidad (tzotzil en Chamula y Zinacantán, tzeltal en Amatenango del Valle), con la finalidad de que los entrevistados comprendieran cada uno de los apartados y, de ser necesario, se aplicará completamente el instrumento en su lengua materna. Por ejemplo, en la sección de síntomas de IAP, previamente se le explicó su definición y posteriormente se le cuestionó sobre los síntomas que ha presentado.

La información se procesó en dos fases. En la primera se realizó un análisis de frecuencias por SP para las principales variables socioeconómicas y productivas (Cuadro I). En la segunda se trató de identificar la relación de las variables socioeconómicas-productivas y el uso de plaguicidas, con las variables de conocimientos y conductas y de IAP. Se utilizaron principalmente pruebas de chi cuadrada para las variables nominales, así como ANOVAy pruebas de Tukey para las variables cuantitativas (Gómez-Gómez et al. 2013). Para este fin se categorizaron las variables relacionadas con los conocimientos y conductas, calificando con un punto las respuestas congruentes con conocimientos y conductas correctas, y con 0 puntos las que se encontraran en el caso contrario. Según las estimaciones obtenidas en la valoración global, se establecieron los puntos de corte a partir del cálculo de los cuartiles para cada conjunto de ítems de cada variable (Triola 2004). Debido a que el conjunto de preguntas para identificar los conocimientos y conductas (cinco y 17 preguntas, respectivamente) presentó diversidad en cuanto a respuestas positivas y negativas, se consideró la sumatoria de respuestas mayoritariamente positivas contenidas en el cuartil IV, para considerarlas como conocimientos y conductas suficientes y apropiadas, respectivamente. Con base en lo anterior, se consideró un máximo de cinco puntos (correspondientes a las mismas respuestas positivas) para identificar si los usuarios poseen suficientes conocimientos sobre uso y manejo de plaguicidas; puntajes $\geq 3$ se consideraron indicativos de conocimientos suficientes (cuartil IV), mientras que puntajes $\leq 2$ se vincularon con conocimientos insuficientes. De la misma manera, para identificar las conductas apropiadas e inapropiadas, se consideró un máximo de 17 puntos, en tanto que los puntajes $\geq 13$ se consideraron representativos de conductas apropiadas (cuartil IV) y los $\leq 12$ se consideraron de conductas inapropiadas.

Finalmente, para clasificar los plaguicidas se consideró la propuesta de la Organización Mundial de la Salud (OMS) basada en la peligrosidad o grado de toxicidad aguda, que agrupa a estos compuestos en cuatro clases según su toxicidad expresada en DL50 $(\mathrm{mg} / \mathrm{kg}$ ): clase IA (extremadamente peligrosos), clase IB (altamente peligrosos), clase II (moderadamente peligrosos) y clase III (ligeramente peligrosos). Para México se realizó un ajuste en el nombre de cada

CUADRO I. CATEGORIZACIÓN DE LAS PRINCIPALES VARIABLES INVOLUCRADAS EN EL ESTUDIO

\begin{tabular}{ll}
\hline Variable & Categorización \\
\hline $\begin{array}{l}\text { Tipo de sistema de producción } \\
\text { Escolaridad del jefe de familia }\end{array}$ & Maíz/flores/hortalizas \\
Edad del jefe de familia & No sabe leer ni escribir/sabe leer y escribir \\
Sexo del jefe de familia & $\leq 44$ años/ $\geq 45$ años* \\
Apoyos gubernamentales & Masculino/femenino \\
Inversión de apoyos gubernamentales en & Procampo/Maíz Solidario/Oportunidades/Amanecer \\
la compra de plaguicidas & Sí/no \\
Capacitación técnica & Recibe/no recibe \\
Superficie agrícola sembrada en el último año & $\leq 0.99$ ha/ $\geq 1.00$ ha \\
Tipo de problemas & Insectos/enfermedades/malezas \\
Ciclos de cultivo en el último año & $\geq 3$ ciclos/1 a 2 ciclos \\
Núm. de cultivos por año & $\geq 2$ cultivos/1 cultivo \\
Tipo de plaguicida por ingrediente activo** & Organofosforado/organoclorado/carbamato/bipiridilo/piretroide/etc. \\
Categoría toxicológica (CT) del plaguicida** & CT I/CT II/CT III/CT IV \\
Conocimientos y conductas sobre el uso y & \\
manejo de plaguicidas & Apropiada/inapropiada \\
Daño a la salud (síntomas de IAP) & Número de síntomas*** \\
\hline
\end{tabular}

* Se determinó con base en la etapa reproductiva y laboral de los mexicanos en zonas rurales según el CONAPO (2014) **Se revisó la ficha técnica del producto para determinar su tipo y categoría toxicológica, a partir del nombre comercial proporcionado por el usuario y la observación de los envases en campo (COFEPRIS 2011, INE 2004)

***Síntomas de intoxicación aguda por plaguicidas (IAP): dolor de cabeza, mareo, dolor de estómago, falta de apetito, ojos llorosos, ardor de ojos, fatiga o debilidad excesiva, dolor muscular, flemas, sed excesiva, comezón, resequedad de la garganta, salivación, hormigueo, nerviosismo, calambres, temblores en manos y/o cuerpo, visión borrosa, ardor en piel, diarrea, náusea o vómito, sudoración excesiva, dificultad para respirar, dolor de pecho 
categoría toxicológica (CT) respetando la clasificación de la OMS, por lo que cada una de éstas se denomina CT I, II, III y IV, respectivamente (INE 2004).

\section{RESULTADOS}

Aproximadamente el $75 \%$ de los JF en el sistema de producción hortícola (SPH) y en el sistema de producción florícola (SPF) son menores de 44 años, en comparación con poco más del $50 \%$ en el sistema de producción de maíz (SPM), lo que evidencia las diferencias significativas entre grupos $\left(X^{2}\right.$ de Pearson $=48.50,2 \mathrm{gl}, p=0.001)$. Se registraron niveles de escolaridad significativamente superiores en el SPF y el SPM respecto a SPH $\left(X^{2}\right.$ de Pearson $=16.99,2 \mathrm{gl}$, $p=0.001)$. Resalta que más del $50 \%$ de los JF en SPH no saben leer ni escribir, en contraste con más del $75 \%$ que sí lo hacen en el SPF y el SPM. Las superficies agrícolas son significativamente más pequeñas en SPH y SPF respecto a SPM ( $2 \mathrm{gl} ; p=0.001)$. En el SPM, se reciben significativamente más apoyos gubernamentales $^{1}\left(X^{2}\right.$ de Pearson $=17.73,2 \mathrm{gl}$, $p=0.001)$ que en el SPF y SPH. Destaca el programa Procampo, cuyos apoyos son recibidos por más del $50 \%$ de productores de maíz y hortalizas. El programa Oportunidades beneficia a una proporción similar en los tres sistemas. Pocos productores de maíz y flores reciben el paquete gubernamental denominado Maíz Solidario. El programa Amanecer beneficia a más UD en el SPM. Los recursos provenientes del programa Procampo son frecuentemente invertidos en la compra de plaguicidas (principalmente en SPM y SPF), seguido de Oportunidades y en menor proporción de Amanecer y Maíz Solidario (Cuadro II).

En el SPH se siembran 18 especies de hortalizas: cilantro, nabo, rábano, repollo, lechuga, betabel, acelga, papa, perejil, brócoli, zanahoria, hierbabuena, coliflor, mostaza, chícharo, haba, calabaza y espinaca, predominando las cuatro primeras. En el SPF se cultivan nueves especies de flores en sus diversas variedades: crisantemo, áster, rosa, alstroemeria, clavel, dalia, nube, azucena y agapanto, predominando las tres primeras. En el SPM se siembra maíz una vez al año en condiciones de temporal y en menor proporción con riego. En el SPF los daños están relacionados con algunos insectos: trips (Frankliniella occidentalis), araña roja (Tetranychus urticae), diversos gusanos que atacan la raíz y la parte aérea de la planta, mosquita blanca (Bemisia tabaci) y pulgón (Myzus persicae); las enfermedades están relacionadas con la roya (género Puccinia), pudrición de la planta, cenicilla (Oidium sp.) y marchitamiento. En el SPH los daños más considerables se deben a gusanos que dañan raíces, tallo y hojas de las hortalizas, seguidos de insectos chupadores (mosquita blanca, pulgón y araña roja). Las enfermedades más frecuentes son pudrición y deformación y/o marchitamiento de las plantas. En el SPM es considerable el daño por arvenses y el provocado por insectos, como los gusanos que atacan la raíz (complejo gallina ciega Phyllophaga spp.), tallo y hoja, así como el gorgojo (Sitophilus zeamais), que ataca el grano en almacenamiento.

En el SPF se utiliza la mayor diversidad de plaguicidas en comparación con el SPH y SPM $(3.2 \pm 1.7$, $2.6 \pm 1.0$ y $2.2 \pm 1.6$ productos, respectivamente $(2 \mathrm{gl}$, $p=0.001)$. En total se identificaron 55 ingredientes activos: 46 se utilizan en el SPF, 20 en el SPH y 18 en el SPM (Cuadro III). En el SPF y SPH se utilizan con más frecuencia insecticidas organofosforados y carbamatos de CT I y II, y el fungicida ditiocarbamato de CT IV. En el SPM destaca significativamente el uso de herbicidas del tipo bipiridilo (CT II), clorofenoxi (CT III) y fosfonato (CT IV). El SPF y el SPH se hace un uso significativamente mayor de plaguicidas de CT I $\left(X^{2}=75.2,2 \mathrm{gl}, p=0.001\right)$ en comparación con el SPM.

En el SPF predomina el uso de siete insecticidas de 19 identificados (abamectina, metamidofos, metomilo, paratión metílico, spinozad, terbufos y carbofuran); para el control de enfermedades fúngicas se detectaron 20 productos de los cuales seis son los más utilizados (mancozeb, myclobutanil, triforine, flutriafol, carbendazim y clorotalonil + cymoxanil). En el caso de las arvenses, aunque no es un problema fuerte, se emplea principalmente el paraquat. En el SPH se utilizan principalmente cuatro insecticidas

\footnotetext{
${ }^{1}$ El Programa de Apoyos directos al Campo (Procampo) del gobierno federal otorga un subsidio directo al ingreso del productor: Se proporciona de manera anual desde 1995 y se asigna por superficie agrícola (hectárea) sin importar los rendimientos obtenidos (SAGARPA 2007). El Programa de Desarrollo Humano Oportunidades entrega de manera bimensual desde 2001 un ingreso en efectivo a los hogares rurales más pobres, a condición de que los niños asistan a la escuela y sus miembros acudan al servicio médico y participen en los talleres de salud y nutrición (Villarreal et al. 2009). Por su parte, el gobierno del estado de Chiapas ha brindado atención integral a los adultos mayores mediante la entrega mensual de pensiones y servicios médicos a través del Programa Amanecer; además, ha apoyado a productores de maíz con la distribución de un paquete tecnológico que incluye fertilizantes y plaguicidas sintéticos, así como semillas híbridas mediante el Programa Maíz Solidario (Gobierno del Estado de Chiapas 2006).
} 
CUADRO II. PRINCIPALES CARACTERÍSTICAS DE LAS UNIDADES DOMÉSTICAS INVOLUCRADAS EN EL ESTUDIO

\begin{tabular}{|c|c|c|c|c|}
\hline & $\begin{array}{c}\text { SPM } \\
(\mathrm{n}=258)\end{array}$ & $\begin{array}{c}\text { SPF } \\
(n=149)\end{array}$ & $\begin{array}{c}\text { SPH } \\
(n=116)\end{array}$ & $\begin{array}{l}X^{2} \text { de Pearson, } \mathrm{RV}, \\
\text { gl, valor de } p\end{array}$ \\
\hline \multicolumn{5}{|l|}{ Edad del jefe de familia (\%) } \\
\hline$\leq 44$ años & 56.6 & 73.2 & 74.1 & \multirow{4}{*}{$\begin{array}{c}16.67 ; \\
16.785 \\
2 ; 0.001\end{array}$} \\
\hline$\geq 45$ años & 43.4 & 26.8 & 25.9 & \\
\hline Media (DE) & $44.1( \pm 14.5)$ & $37.0( \pm 11.8)$ & $35.7( \pm 13.9)$ & \\
\hline Rango (años) & $17-83$ & $16-67$ & $14-71$ & \\
\hline \multicolumn{5}{|l|}{ Sexo del jefe de familia (\%) } \\
\hline Masculino & 91.1 & 97.3 & 76.7 & 30.96 \\
\hline Femenino & 8.9 & 2.7 & 23.3 & $\begin{array}{c}29.64 \\
2 ; 0.001\end{array}$ \\
\hline \multicolumn{5}{|l|}{ Escolaridad del jefe de familia (\%) } \\
\hline No sabe leer ni escribir & 22.9 & 21.5 & 55.2 & 46.69 \\
\hline Sabe leer y escribir & 77.1 & 78.5 & 44.8 & $\begin{array}{c}43.68 \\
2 ; 0.000\end{array}$ \\
\hline \multicolumn{5}{|l|}{$\begin{array}{l}\text { Superficie total destinada a la } \\
\text { agricultura en el último año (ha) }\end{array}$} \\
\hline Media (DE) & $1.74( \pm 1.23)^{\mathrm{a}}$ & $0.34( \pm 0.49)^{\mathrm{b}}$ & $0.38( \pm 0.58)^{\mathrm{b}}$ & \\
\hline Rango (ha) & $0.25-8.0$ & $0.002-3.16$ & $0.010-4.40$ & \\
\hline $\begin{array}{l}\text { Apoyos de programas } \\
\text { gubernamentales que recibe la } \\
\text { unidad doméstica / (inversión en } \\
\text { la compra de plaguicidas) (\%) }\end{array}$ & $68.3(46.0)$ & $73.1(41.0)$ & $61.2(23.3)$ & $\begin{array}{c}13.74 ; \\
13.27 \\
2 ; 0.001\end{array}$ \\
\hline Procampo & $53.5(43.7)$ & $36.2(33.6)$ & $6.9(6.0)$ & \\
\hline Oportunidades & $60.1(5.0)$ & $62.4(8.1)$ & $56.9(16.4)$ & \\
\hline Amanecer & $8.5(2.0)$ & $1.3(0.7)$ & $2.6(1.7)$ & \\
\hline Maíz solidario & $3.1(2.7)$ & $3.4(3.4)$ & - & \\
\hline
\end{tabular}

Letras distintas son estadísticamente diferentes $(\alpha=0.05)$

SPM: sistema de producción de maíz; SPF: sistema de producción de flores; SPH: sistema de producción de hortalizas

CUADRO III. USO DE PLAGUICIDAS POR CATEGORÍA TOXICOLÓGICA

\begin{tabular}{rccccc}
\hline & \multicolumn{4}{c}{ Utilización de plaguicidas por categoría toxicológica (CT) } \\
\cline { 2 - 6 } & CT I & CT II & CT III & CT IV & Las 4 CT \\
\hline SPM ( \% de UD usuarias) & 46.9 & 59.0 & 57.8 & 47.3 & 100.0 \\
Núm. de IA & 3 & 3 & 6 & 6 & 18 \\
Promedio de IA por UD & $0.5( \pm 0.7)^{\mathrm{a}}$ & $0.6( \pm 0.7)^{\mathrm{a}}$ & $0.6( \pm 0.7)^{\mathrm{a}}$ & $0.5( \pm 0.6)^{\mathrm{a}}$ & $2.2( \pm 1.6)^{\mathrm{a}}$ \\
SPF (\% de UD usuarias) & 79.2 & 51.7 & 42.3 & 56.4 & 100.0 \\
Núm. de IA & 6 & 7 & 10 & 23 & 46 \\
Promedio de IA por UD & $1.3( \pm 1.0)^{\mathrm{b}}$ & $0.6( \pm 0.7)^{\mathrm{a}}$ & $0.5( \pm 0.7)^{\mathrm{a}}$ & $0.8( \pm 0.9)^{\mathrm{b}}$ & $3.2( \pm 1.7)^{\mathrm{b}}$ \\
SPH (\% de UD usuarias) & 87.1 & 30.2 & 7.8 & 85.3 & 100.0 \\
Núm. de IA & 4 & 3 & 4 & 9 & 20 \\
Promedio de IA por UD & $1.1( \pm 0.6)^{\mathrm{c}}$ & $0.3( \pm 0.6)^{\mathrm{b}}$ & $0.1( \pm 0.3)^{\mathrm{b}}$ & $1.2( \pm 0.7)^{\mathrm{c}}$ & $2.6( \pm 1.0)^{\mathrm{c}}$ \\
\% total de UD usuarias (n=523) & 65.0 & 50.5 & 42.3 & 58.3 & 100.0 \\
\hline
\end{tabular}

Categoría toxicológica: CT I, extremadamente peligroso; CT II, altamente peligroso; CT III, moderadamente peligroso; CT IV, ligeramente peligroso

SPM: sistema de producción de maíz $(n=258)$; SPF: sistema de producción de flores $(\mathrm{n}=149)$; SPH: sistema de producción de hortalizas $(\mathrm{n}=116)$

UD: unidad doméstica; IA: ingrediente activo

Letras distintas son estadísticamente diferentes $(\alpha=0.05)$ 
de 11 identificados (metamidofos, paration metílico, carbofuran y foxim); la prevención, control y combate de las enfermedades fúngicas se realiza mayoritariamente con mancozeb, de seis fungicidas identificados (el herbicida paraquat es poco utilizado, ya que los ciclos agrícolas son cortos y la limpia se realiza de manera manual). En el SPM el crecimiento de arvenses es un problema muy fuerte, por lo cual se utilizan principalmente tres herbicidas de cinco identificados, paraquat, 2-4 D y glifosato, así como cuatro insecticidas de 10 identificados: paratión metílico, fosfuro de aluminio, monocrotofos y lambda cyhalotrina (Cuadro IV).

En los tres sistemas prácticamente no se ha proporcionado asistencia y/o capacitación formal en el manejo de plaguicidas. En este sentido, es notoria la ausencia de intervención gubernamental y de las empresas dedicadas a la venta y distribución de dichos productos. La mayoría de los usuarios ha aprendido a utilizarlos a través de sus familiares, vecinos, amigos y de la observación. Aproximadamente el $50 \%$ de los productores en el SPF y SPM no leen la etiqueta, a pesar de que la mayor parte de los JF cuentan con una escolaridad mayor con relación a los productores de hortalizas. En los tres sistemas, la mayoría de los individuos desconoce el significado de los colores de las etiquetas, independientemente de su escolaridad. La mayoría de los campesinos en el SPM y SPF mencionaron seguir las dosis indicadas en las instrucciones de los productos empleados (Cuadro V). De acuerdo con lo anterior, en el SPH se observaron los puntajes de conocimientos apropiados estadísticamente significativos más bajos en comparación con el SPM y el SPF $(1.1 \pm 1.1 ; 1.8 \pm$ 1.1 y $2.0 \pm 1.3$, respectivamente; $2 \mathrm{gl} ; p=0.001$ ), relacionados con su baja escolaridad $(r=0.263$, $p=0.001)$. Por su parte, los productores de maíz, no obstante que presentaron una escolaridad más alta, tuvieron los puntajes de conductas apropiadas más bajos respecto al SPH y SPF $(10.2 \pm 1.8,11.2 \pm 1.9$ y $11.6 \pm 1.6$, respectivamente; $2 \mathrm{gl} ; p=0.000$ ). A pesar de las diferencias significativas e independientemente de la escolaridad, se observó que la mayor parte de la población en los tres SP posee conocimientos insuficientes $(87.1 .0,69.4$ y $62.4 \%$ para SPH, SPM y SPF, respectivamente; $X^{2}=20.3, \mathrm{RV}=22.4,2 \mathrm{gl}$, $p=0.000$ ), además de conductas inapropiadas respecto del uso y manejo de plaguicidas $(90.3,75.8$ y $70.7 \%$ para SPM, SPF y SPH respectivamente $\left(X^{2}=25.8, \mathrm{RV}=26.4,2 \mathrm{gl}, p=0.001\right)$.

$\mathrm{Al}$ analizar las conductas de uso, manejo y almacenamiento de plaguicidas (Cuadro VI) se observa que la mayoría de los campesinos no se protege al preparar las mezclas y aplicar los productos; quienes sí lo hacen utilizan principalmente bolsas de plástico para proteger sus manos al diluir el agroquímico con agua. Hay mayor descuido en la preparación en el SPM, donde se manifiestan salpicaduras o derrames. Los campesinos utilizan su ropa cotidiana para realizar las labores agrícolas; aquellos que manifiestan protegerse emplean camisa de manga larga, pantalón de mezclilla y botas de plástico. Son pocos los que usan pañuelo para protegerse la boca y bolsas de plástico para cubrir manos y espalda. En el caso de las mujeres, específicamente en el $\mathrm{SPH}$, su vestimenta consta de blusa de manga corta acompañada ocasionalmente de suéter, falda de lana de borrego y sandalias de plástico; son pocas las que utilizan ocasionalmente botas de plástico. La mayoría de los usuarios mencionó lavarse las manos para ingerir sus alimentos después de preparar y/o aplicar los plaguicidas, así como bañarse después de la jornada laboral y cambiarse de ropa. Es frecuente el consumo de alimentos a un costado de la parcela en el SPM. La ropa de trabajo se lava el mismo día que se utilizó; sin embargo, hay un grupo de JF que lava la ropa sucia una o dos veces por semana. Se detectó a usuarios, sobre todo en el SPM y SPF, que duermen con la ropa de trabajo cuando se quedan en las parcelas. El lavado de ropa sucia está bajo la responsabilidad de la mujer (esposa, hija, hermana o madre del usuario); son pocos los hombres que manifestaron lavar ellos mismos su ropa. En el caso del SPH, las mujeres que son JF (23.3 \%) son las responsables de las labores agrícolas además de cumplir con funciones domésticas (lavado de su ropa sucia y la de su familia). La mayoría de los JF manifestó que su bomba de aspersión se encontraba en buen estado; sin embargo, aquellos que mencionaron algún daño a su equipo lo seguían utilizando a pesar de los desperfectos. Es frecuente que los dispositivos en buen estado se tapen y que los usuarios los arreglen introduciendo la mano e, incluso, utilizando la boca (SPM). Aproximadamente $80 \%$ de los usuarios en cada sistema lavan su equipo; aquellos que no lo hacen mencionan que no es necesario debido a que serán utilizados nuevamente.

En general, la fumigación se realiza en diferentes horarios y sin previo aviso a los vecinos cuyas viviendas se encuentran próximas a las áreas rociadas. En el SPH los usuarios asperjan sus parcelas con más frecuencia en condiciones de viento. Son pocos los JF que manifestaron la presencia de niños al fumigar la parcela; sin embargo, les están enseñando a utilizar los plaguicidas, principalmente en el SPM y SPF. En el SPM estos compuestos se guardan con más 
CUADRO IV. INGREDIENTES ACTIVOS DE PLAGUICIDAS POR CATEGORÍA TOXICOLÓGICA IDENTIFICADOS EN EL ESTUDIO

\begin{tabular}{|c|c|c|c|c|c|c|c|c|c|}
\hline Ingrediente activo & $\begin{array}{l}\text { Clasificación } \\
\text { química }\end{array}$ & Tipo $^{1}$ & $\mathrm{CT}$ & $\begin{array}{l}\text { SPM } \\
(\mathrm{n}= \\
258) \\
\%\end{array}$ & $\begin{array}{l}\text { SPF } \\
(\mathrm{n}= \\
149) \\
\%\end{array}$ & $\begin{array}{l}\text { SPH } \\
(\mathrm{n}= \\
116) \\
\%\end{array}$ & Nombres comerciales & $\begin{array}{l}\text { Lista } \\
\text { PAP }^{4}\end{array}$ & $\begin{array}{l}\text { Núm. de } \\
\text { países con } \\
\text { prohibición }{ }^{5}\end{array}$ \\
\hline Abamectina $^{4}$ & Pentaciclina & $\mathrm{I}$ & I & & 43.0 & - & Agrimec & 1,3 & \\
\hline $\begin{array}{l}\text { Fosfuro de } \\
\text { aluminio }^{4}\end{array}$ & Fosfamina & I & I & 23.6 & - & - & Fosfuro de aluminio & 1,3 & 1 \\
\hline Etoprófos $^{4}$ & Organofosforado & $\mathrm{I}$ & $\mathrm{I}$ & - & 5.4 & 4.3 & Mocap & 1,2 & 8 \\
\hline Metamidófos ${ }^{2,4}$ & Organofosforado & $\mathrm{I}$ & $\mathrm{I}$ & - & 38.9 & 81.9 & $\begin{array}{l}\text { Metrifos, Monitor } 600 \text {, } \\
\text { Tamaron }\end{array}$ & $1,3,4$ & 20 \\
\hline Monocrotofos ${ }^{4}$ & Organofosforado & I & I & 8.9 & 0.7 & - & Nuvacrón, Vanucron & $1,3,4$ & 30 \\
\hline Paration metílico ${ }^{4}$ & Organofosforado & $\mathrm{I}$ & $\mathrm{I}$ & 24.0 & 25.5 & 16.4 & Paration metílico, Foley & 1,4 & \\
\hline Terbufos ${ }^{4}$ & Organofosforado & $\mathrm{I}$ & $\mathrm{I}$ & - & 12.1 & 3.5 & Anater, Coster, Counter & 1 & 7 \\
\hline Cadusafos $^{4}$ & Organofosforado & $\mathrm{I}$ & II & - & 0.7 & - & Rugby & 1,3 & 4 \\
\hline Isazofos & Organofosforado & $\mathrm{I}$ & II & - & 0.7 & - & Triunfo & & \\
\hline Ometoato $^{4}$ & Organofosforado & I & II & - & 6.7 & 2.6 & Folimat & $1,2,3$ & 3 \\
\hline Tiodicarb $^{4}$ & Carbamato & I & II & 0.8 & - & - & Semevin & 2,3 & 2 \\
\hline Carbofuran $^{4}$ & Carbamato & $\mathrm{I}$ & II & 1.6 & 8.1 & 11.2 & Cufuran, Furadan & $1,3,4$ & 19 \\
\hline Metomilo $^{4}$ & Carbamato & I & II & - & 31.5 & - & Lannate & 1,3 & 11 \\
\hline Endosulfam & Organoclorado & $\mathrm{I}$ & II & - & 5.4 & - & Thionex, Thiodan & 1,4 & 44 \\
\hline Paraquat $^{2,4}$ & Bipiridilo & $\mathrm{H}$ & II & 69.4 & 8.7 & 20.7 & $\begin{array}{l}\text { Chamusquat, Cuproquat, } \\
\text { Garraquat, Gramocil, } \\
\text { Gramoxone, Gramuron, } \\
\text { Secaduro, Diabloquat, } \\
\text { Paraquat }\end{array}$ & 1 & 8 \\
\hline $2-4 D^{4}$ & $\begin{array}{l}\text { Clorofenoxi (ácido } \\
\text { fenoxiacético) }\end{array}$ & $\mathrm{H}$ & III & 59.3 & 2.0 & - & $\begin{array}{l}\text { Arrasador, Herbidex, Amina, } \\
\text { Esteron } 47 \text {, Herbipol }\end{array}$ & 1 & 2 \\
\hline Clethodim & Ciclohexanodiona & $\mathrm{H}$ & III & - & 3.4 & - & Cedrus & & \\
\hline Clorpirifos etil $^{4}$ & Organofosforado & I & III & - & 4.7 & - & Lorsban & 3 & 1 \\
\hline Permetrina $^{4}$ & Piretroide & $\mathrm{I}$ & III & - & - & 3.4 & Ambush & 2,3 & 2 \\
\hline $\begin{array}{l}\text { Cipermetrina }{ }^{4} \\
+ \text { dimetoato }^{4}\end{array}$ & Piretroide & I & III & 0.4 & - & - & Cipertoato & $3 ; 3$ &,- 4 \\
\hline Cipermetrina $^{4}$ & Piretroide & $\mathrm{I}$ & III & 2.7 & - & 1.7 & Arrivo, Gallo & 3 & \\
\hline Deltametrina $^{4}$ & Piretroide & $\mathrm{I}$ & III & 0.8 & 1.3 & 2.6 & Decis, Deltametrina, Butox & 2,3 & \\
\hline $\begin{array}{l}\text { Lambda } \\
\text { cyhalotrina }\end{array}$ & Piretroide & $\mathrm{I}$ & III & 9.3 & 1.3 & - & Karate, Pateador, Pulsar & $1,2,3$ & \\
\hline Spinozad $^{3,4}$ & Sin clasificación & $\mathrm{I}$ & III & - & 18.1 & - & Spintor & 3 & \\
\hline Miclobutanil & Triazol & $\mathrm{F}$ & III & - & 12.8 & - & Rally & & \\
\hline Propiconazol & $\begin{array}{l}\text { Triazol más } \\
\text { anilinopirimidina }\end{array}$ & $\mathrm{F}$ & III & - & 3.4 & - & Tilt & & \\
\hline Tiabendazol & Benzimidazol & $\mathrm{F}$ & III & - & 4.0 & - & Tecto & & 1 \\
\hline $\begin{array}{l}\text { Metaldehido } \\
\text { con metomil y } \\
\text { methiocarb }\end{array}$ & $\begin{array}{l}\text { Metaldehído (adehído) } \\
\text { metomil y methicarb } \\
\text { (carbamatos, CT II) }\end{array}$ & $\mathrm{M}, \mathrm{I}$ & III & 0.4 & 0.7 & 0.9 & Caracolex & $\begin{array}{l}1,3 \\
1,3\end{array}$ & $-, 11,3$ \\
\hline Atrazina $^{4}$ & Triazina & $\mathrm{H}$ & IV & 0.8 & 0.7 & - & Gesaprim, revólver & 2 & 10 \\
\hline
\end{tabular}

${ }^{1}$ Tipo: I, insecticida; H, herbicida; F, fungicida; M, molusquicida; B, bactericida

${ }^{2}$ Uso restringido (COFEPRIS 2011)

${ }^{3}$ Sin clasificación (INE 2004)

${ }^{4}$ Criterios de inclusión en la lista de plaguicidas altamente peligrosos de PAN Internacional (2015a): (1) toxicidad aguda alta; (2) efectos crónicos en la salud humana; (3) toxicidad ambiental, y (4) restringidos o prohibidos por convenios ambientales

${ }^{5}$ Lista de plaguicidas prohibidos en otros países de PAN International (2015b)

SPM: sistema de producción de maíz, SPF: sistema de producción de flores, SPH: sistema de producción de hortalizas 
CUADRO IV. INGREDIENTES ACTIVOS DE PLAGUICIDAS POR CATEGORÍA TOXICOLÓGICA IDENTIFICADOS EN EL ESTUDIO

\begin{tabular}{|c|c|c|c|c|c|c|c|c|c|}
\hline Ingrediente activo & $\begin{array}{l}\text { Clasificación } \\
\text { química }\end{array}$ & Tipo $^{1}$ & $\mathrm{CT}$ & $\begin{array}{l}\text { SPM } \\
(\mathrm{n}= \\
258) \\
\%\end{array}$ & $\begin{array}{l}\text { SPF } \\
(\mathrm{n}= \\
149) \\
\%\end{array}$ & $\begin{array}{l}\text { SPH } \\
(\mathrm{n}= \\
116) \\
\%\end{array}$ & Nombres comerciales & $\begin{array}{l}\text { Lista } \\
\text { PAP }^{4}\end{array}$ & $\begin{array}{l}\text { Núm. de } \\
\text { países con } \\
\text { prohibición }\end{array}$ \\
\hline Glifosato & $\begin{array}{l}\text { Fosfonometilglicina } \\
\text { (fosfonato) }\end{array}$ & $\mathrm{H}$ & IV & 49.6 & 1.3 & 4.3 & $\begin{array}{l}\text { Coloso, Glifosato, Diablozato, } \\
\text { Rival, Faena, Secafin, Takle, } \\
\text { Glifos }\end{array}$ & 2 & 1 \\
\hline $\begin{array}{l}\text { Glufosinato de } \\
\text { amonio }^{4}\end{array}$ & Organofosforado & $\mathrm{H}$ & IV & 0.4 & - & - & Finale & 2 & \\
\hline Cyromacina & Triazina & I & IV & - & 2.7 & - & Trigard & & \\
\hline Flufenoxuron $^{4}$ & Benzoilurea & I & IV & - & 0.7 & 0.9 & Cascade & 3 & 1 \\
\hline Foxim & Organofosforado & I & IV & 0.8 & - & 10.3 & Volaton & & \\
\hline Azufre elemental & Inorgánico & I y F & IV & - & 4.0 & - & Sultron & & \\
\hline Fosetil-Al & $\begin{array}{l}\text { Alcoil fosfonato } \\
\text { (fosfonato) }\end{array}$ & $\mathrm{F}$ & IV & - & 2.0 & - & Aliette WDG & & \\
\hline Flutriafol & Triazol & $\mathrm{F}$ & IV & - & 4.7 & 一 & Impact & & \\
\hline Azoxistrobin & $\begin{array}{l}\text { Pirimidina } \\
\text { (estrobilurina }+ \\
\text { triazol) }\end{array}$ & F & IV & - & 3.4 & 5.2 & Amistar & & \\
\hline Captan & Carboxamida & $\mathrm{F}$ & IV & - & 2.0 & - & Captan & & 6 \\
\hline Carbendazim $^{4}$ & Benzimidazol & $\mathrm{F}$ & IV & - & 4.7 & - & Derosal, Prozycar & 2 & 2 \\
\hline Cymoxanil & $\begin{array}{l}\text { Sal inorgánica de } \\
\text { cobre }\end{array}$ & $\mathrm{F}$ & IV & - & - & 3.4 & Curzate & & \\
\hline$\underline{\text { Dicloran }}$ & Nitroanilina & $\mathrm{F}$ & IV & 一 & 0.7 & - & Botran & & 1 \\
\hline $\begin{array}{l}\text { Dimeticorf }+ \\
\text { mancozeb }^{4}\end{array}$ & Morfolina & $\mathrm{F}$ & IV & 0.4 & - & - & Acrobat & 1,3 &,- 1 \\
\hline Kresoxim metil $^{4}$ & $\begin{array}{l}\text { Metoximinoacetato de } \\
\text { estrobilurina }\end{array}$ & $\mathrm{F}$ & IV & - & 4.0 & - & Stroby & 2 & \\
\hline Mancozeb $^{4}$ & Ditiocarbamato & $\mathrm{F}$ & IV & - & 26.8 & 81.0 & Manzate, Pol-zeb 80, Ridomil & 2 & 1 \\
\hline Mandipropamida $^{3}$ & Sin clasificación & $\mathrm{F}$ & IV & - & 0.7 & - & Revus & & \\
\hline $\begin{array}{l}\text { Propamocarb } \\
\text { clorhidrato }\end{array}$ & Carbamato & F & IV & - & 2.7 & 6.0 & Previcur & & \\
\hline $\begin{array}{l}\text { Propamocarb } \\
\text { clorhidrato } \\
+ \text { fenamidona } \\
\end{array}$ & $\begin{array}{l}\text { Carbamato } \\
\text { (propamocarb); } \\
\text { imidazol (fenamidona) }\end{array}$ & $\mathrm{F}$ & IV & - & 1.3 & 0.0 & Consento/Fenora & & \\
\hline Tebuconazole & Benzimidazol & $\mathrm{F}$ & IV & - & 1.3 & 0.9 & Folicur & & \\
\hline Tiofanato metílico ${ }^{4}$ & Tiocarbamato & $\mathrm{F}$ & IV & - & 1.3 & - & Prontius & 2 & \\
\hline Trifloxystrobin & Estrobilurinas & $\mathrm{F}$ & IV & 一 & 4.7 & 一 & Flint & & \\
\hline Triforine & Piperazina & $\mathrm{F}$ & IV & - & 7.4 & - & Saprol & & \\
\hline $\begin{array}{l}\text { Clorotalonil }{ }^{2,4} \\
+ \text { cimoxanil } \\
\end{array}$ & $\begin{array}{l}\text { Aromático } \\
\text { policlorado }\end{array}$ & $\mathrm{F}$ & IV & - & 4.7 & 2.6 & $\begin{array}{l}\text { Bravo 720, Dragonil, Leal } \\
800, \text { Strike }\end{array}$ & 1,2 & $2,-$ \\
\hline Quintozeno $^{2}$ & Clorobenceno & $\mathrm{F}$ & IV & 0.4 & 0.7 & - & Interguzan, Controller & & 11 \\
\hline Oxitetraciclina & Antibiótico & B & IV & - & 0.7 & - & Terramicina & & \\
\hline
\end{tabular}

${ }^{1}$ Tipo: I, insecticida; $\mathrm{H}$, herbicida; F, fungicida; M, molusquicida; B, bactericida

${ }^{2}$ Uso restringido (COFEPRIS 2011)

${ }^{3}$ Sin clasificación (INE 2004)

${ }^{4}$ Criterios de inclusión en la lista de plaguicidas altamente peligrosos de PAN Internacional (2015a): (1) toxicidad aguda alta; (2) efectos crónicos en la salud humana; (3) toxicidad ambiental, y (4) restringidos o prohibidos por convenios ambientales

${ }^{5}$ Lista de plaguicidas prohibidos en otros países de PAN International (2015b)

SPM: sistema de producción de maíz, SPF: sistema de producción de flores, SPH: sistema de producción de hortalizas 
CUADRO V. RESPUESTAS POSITIVAS A LAS PREGUNTAS PARA IDENTIFICAR LOS CONOCIMIENTOS CONSIDERADOS COMO SUFICIENTES RELACIONADOS CON EL USO Y MANEJO DE PLAGUICIDAS

\begin{tabular}{|c|c|c|c|c|}
\hline Pregunta & $\begin{array}{c}\text { SPM } \\
(\mathrm{n}=258) \\
\%\end{array}$ & $\begin{array}{c}\text { SPH } \\
(\mathrm{n}=116) \\
\%\end{array}$ & $\begin{array}{c}\text { SPF } \\
(\mathrm{n}=149) \\
\%\end{array}$ & $\begin{array}{c}\text { Total } \\
(\mathrm{n}=523) \\
\%\end{array}$ \\
\hline 1. ¿Ha recibido capacitación? & 3.1 & 5.2 & 8.7 & 5.2 \\
\hline \multicolumn{5}{|l|}{ 2. ¿Quién le ha enseñado a utilizar plaguicidas? } \\
\hline Familiares & 60.8 & 51.7 & 34.3 & 51.2 \\
\hline Vecinos y/o amigos & 13.6 & 16.3 & 29.5 & 18.7 \\
\hline Observación & 17.5 & 17.3 & 20.8 & 18.4 \\
\hline Vendedor y/o técnico de la tienda comercial & 6.9 & 13.0 & 13.5 & 10.1 \\
\hline No contestó & 1.2 & 1.7 & 1.9 & 1.6 \\
\hline 3. ¿El vendedor le dice cómo utilizarlo? & 59.2 & 47.4 & 47.0 & 53.1 \\
\hline 4. ¿Leyó la etiqueta la primera vez que compró o utilizó el plaguicida? & 52.0 & 27.7 & 57.1 & 48.1 \\
\hline 5. ¿Sabe qué significan los colores de las etiquetas? & 10.2 & 2.6 & 15.4 & 10.0 \\
\hline 6. ¿Sigue las dosis indicadas en las instrucciones al preparar la mezcla? & 54.7 & 23.3 & 69.8 & 52.0 \\
\hline
\end{tabular}

SPM: sistema de producción de maíz, SPF: sistema de producción de flores, SPH: sistema de producción de hortalizas

frecuencia en el interior de las viviendas. En el SPF y SPH se observó con más frecuencia la utilización de bodegas exclusivas para el almacenamiento de dichos insumos fuera de la vivienda y en la parcela. La mayor parte de los usuarios quema los envases vacíos; otros los tiran a la basura, los abandonan en la parcela, los entierran o los reutilizan principalmente para el almacenamiento de agua. Una fracción de los usuarios no respondió esta pregunta.

Los productores manifestaron diversos síntomas de IAP después de fumigar sus parcelas. Sólo el dolor de cabeza fue significativo para las diferentes CT (CT I: $X^{2}=3.92, \mathrm{RV}=3.915,1 \mathrm{gl}, p=$ 0.048 ; CT II, III y IV: $X^{2}=10.75, \mathrm{RV}=10.69,1 \mathrm{gl}$, $p=0.001$; todas las CT: $X^{2}=18.77, \mathrm{RV}=19.16$, $1 \mathrm{gl}, p=0.000)$. Aunque los demás síntomas no fueron significativos, se observa que una proporción considerable de productores de los diferentes sistemas manifestó algún tipo de IAP después de emplear los diferentes productos, siendo los floricultores los que mencionaron más molestias, seguidos de los horticultores y los productores de maíz $(77.9,65.5$ y $52.7 \%$, respectivamente). Casi $50 \%$ de los floricultores manifestaron por lo menos cuatro síntomas de IAP, en comparación con el $20 \%$ de los productores de hortalizas y maíz. El principal síntoma fue dolor de cabeza, seguido de problemas respiratorios (resequedad de la garganta, flemas, dificultad para respirar, dolor en el pecho), problemas visuales (irritación, ardor, lagrimeo, visión borrosa), náusea/mareos/vómitos, y en menor proporción dolor abdominal y problemas musculoesqueléticos (principalmente fatiga/debilidad, dolor muscular y nerviosismo). La proporción de casos con síntomas de IAP por CT I en los productores de maíz se incrementó casi un $100 \%$ con el uso de IA de CT II, III y IV, en comparación con los floricultores y horticultores, en quienes no se aprecia este fenómeno. En estos últimos la proporción de casos fue muy similar entre las diferentes CT (Fig. 2).

\section{DISCUSIÓN}

A pesar de las diferencias significativas entre los tres sistemas estudiados, los resultados obtenidos en el presente estudio indican de manera general una alta dependencia del uso de plaguicidas. La justificación del empleo de insecticidas y fungicidas en el SPF y SPH radica en el fuerte desequilibrio de los agroecosistemas, provocado por el uso intensivo de pequeñas superficies agrícolas donde se siembran especies vegetales de ciclo corto varias veces al año; incluso es probable que, al incrementarse las superficies agrícolas, también aumente el uso de dichos insumos. Este proceso de intensificación a lo largo de los años ha provocado que los cultivos sean vulnerables al ataque de insectos y enfermedades fúngicas, por lo que su prevención y control se realiza mediante la aplicación de los plaguicidas identificados, de manera similar a lo reportado por Montoro et al. (2009) para Perú. Por su parte, la justificación del uso de herbicidas (con mayor frecuencia en el cultivo de maíz) radica en el control de arvenses como principal problema, ya que como indican Guillén et al. (2004) estos compuestos disminuyen la carga de trabajo y el tiempo de las labores de limpieza agrícolas, pues las superficies en el SPM son mucho más grandes en comparación con el SPF y SPH. 
CUADRO VI. RESPUESTAS POSITIVAS A LAS PREGUNTAS PARA CONOCER LAS CONDUCTAS APROPIADAS EN EL USO, MANEJO Y ALMACENAMIENTO DE PLAGUICIDAS

\begin{tabular}{|c|c|c|c|c|}
\hline Pregunta & $\begin{array}{c}\text { SPM } \\
(\mathrm{n}=258) \\
\%\end{array}$ & $\begin{array}{c}\text { SPH } \\
(n=116) \\
\%\end{array}$ & $\begin{array}{c}\text { SPF } \\
(\mathrm{n}=149) \\
\%\end{array}$ & $\begin{array}{c}\text { Total } \\
(\mathrm{n}=523) \\
\%\end{array}$ \\
\hline $\begin{array}{l}\text { 1. ¿Utiliza alguna protección para sus manos al momento de preparar la } \\
\text { mezcla de plaguicidas? ¿Qué utiliza? }\end{array}$ & 28.0 & 31.9 & 43.0 & 33.1 \\
\hline Bolsa de plástico & 14.9 & 28.4 & 27.6 & 21.5 \\
\hline Guantes & 13.1 & 3.5 & 15.4 & 11.6 \\
\hline \multicolumn{5}{|l|}{ 2. ¿Qué utiliza como equipo de protección al fumigar? } \\
\hline Pañuelo para cubrirse la boca o cubrebocas & 7.8 & 3.3 & 10.7 & 7.6 \\
\hline Mascarilla & 0.4 & -- & 2.0 & 0.8 \\
\hline Guantes o bolsa de plástico & 3.5 & 6.1 & 4.7 & 4.4 \\
\hline Impermeable o bolsa de plástico & 0.4 & 1.7 & 3.4 & 1.5 \\
\hline 3. ¿Cuando prepara la mezcla, se derrama o salpica? & 50.8 & 25.0 & 33.0 & 40.0 \\
\hline $\begin{array}{l}\text { 4. Después de preparar o aplicar los plaguicidas, ¿se lava las manos antes } \\
\text { de comer, fumar o realizar otra actividad? }\end{array}$ & 97.4 & 96.5 & 97.3 & 97.2 \\
\hline 5. Después de aplicar los plaguicidas, ¿se baña? & 97.4 & 98.2 & 93.9 & 96.6 \\
\hline 6. ¿Se cambia de ropa después de aplicar los plaguicidas? & 96.2 & 97.4 & 94 & 95.8 \\
\hline $\begin{array}{l}\text { 7. Cuando aplica los plaguicidas, ¿come o bebe en el interior de la parcela } \\
\text { o a un costado de ésta? }\end{array}$ & 61.5 & 13.0 & 22.8 & 39.7 \\
\hline \multicolumn{5}{|l|}{ 8. ¿Con qué frecuencia lava la ropa que utilizó para fumigar? } \\
\hline El mismo día que la utilizó & 72.9 & 83.6 & 79.2 & 77.1 \\
\hline Una o dos veces por semana & 26.1 & 15.5 & 19.4 & 21.8 \\
\hline No contestó & 1.0 & 0.9 & 1.4 & 1.1 \\
\hline ¿La lava usted? & 1.9 & 5.1 & 23.3 & 8.8 \\
\hline 9. ¿Duerme con la ropa de trabajo? & 10.9 & 3.5 & 8.0 & 8.4 \\
\hline \multicolumn{5}{|l|}{ 10. ¿Está en buen estado su bomba de aspersión? ¿Cuando se tapa qué } \\
\hline hace? & 86.3 & 92.2 & 85.2 & 87.3 \\
\hline Utiliza la mano sin algún tipo de protección & 55.8 & 79.3 & 46.3 & 58.3 \\
\hline Utiliza la boca & 3.1 & -- & -- & 1.5 \\
\hline $\begin{array}{l}\text { 11. Cuando termina de aplicar los plaguicidas, ¿lava el equipo de } \\
\text { aspersión? }\end{array}$ & 77.1 & 79.3 & 75.1 & 77.0 \\
\hline 12. ¿Avisa a sus vecinos cuando va a fumigar? & 10.2 & 12.9 & 17.5 & 12.9 \\
\hline 13. Cuando hace mucho viento, ¿aplica los plaguicidas? & 13.9 & 36.2 & 22.8 & 21.4 \\
\hline 14. Cuando aplica los plaguicidas, ¿hay niños presentes? & 7.0 & 6.1 & 2.7 & 5.6 \\
\hline 15. ¿Está enseñando a sus hijos a utilizar los plaguicidas? & 48.8 & 34.5 & 52.4 & 46.7 \\
\hline \multicolumn{5}{|l|}{ 16. ¿Dónde guarda los plaguicidas? } \\
\hline Interior de su casa (cuarto, rincón, ropero, tapanco) & 39.2 & 16.4 & 22.1 & 29.3 \\
\hline Exterior de su casa (corredor, colgado) & 27.2 & 20.7 & 13.1 & 21.9 \\
\hline Bodega exclusiva para los plaguicidas & 33.6 & 62.9 & 64.8 & 48.8 \\
\hline \multicolumn{5}{|l|}{ 17. ¿Qué hace con los envases vacíos? } \\
\hline Los quema & 55.5 & 90.5 & 45.7 & 60.5 \\
\hline Los tira a la basura & 21.3 & 6.9 & 24.9 & 19.1 \\
\hline Los abandona en la parcela & 20.6 & 1.7 & 5.0 & 12.0 \\
\hline Los reutiliza & 0.8 & 0.9 & 0.7 & 0.8 \\
\hline Los entierra & 1.2 & -- & 1.4 & 1.0 \\
\hline No contestó & 0.6 & 0.0 & 22.3 & 6.6 \\
\hline
\end{tabular}

SPM: sistema de producción de maíz, SPF: sistema de producción de flores, SPH: sistema de producción de hortalizas

Los principales grupos químicos y principios activos identificados en los tres SP son similares a los reportados por González-Arías et al. (2010) para el estado de Nayarit, México. El uso de plaguicidas en el SPF (principalmente organofosforados y ditiocarbamatos) coincide con lo reportado por Varona et al. (2005) para comunidades florícolas de Colombia, con la diferencia de que los productos más utilizados en esas comunidades son los ditiocarbamatos y organoclorados, y en menor proporción los organofosforados. Los herbicidas identificados en el SPM son similares en las zonas agrícolas del norte 

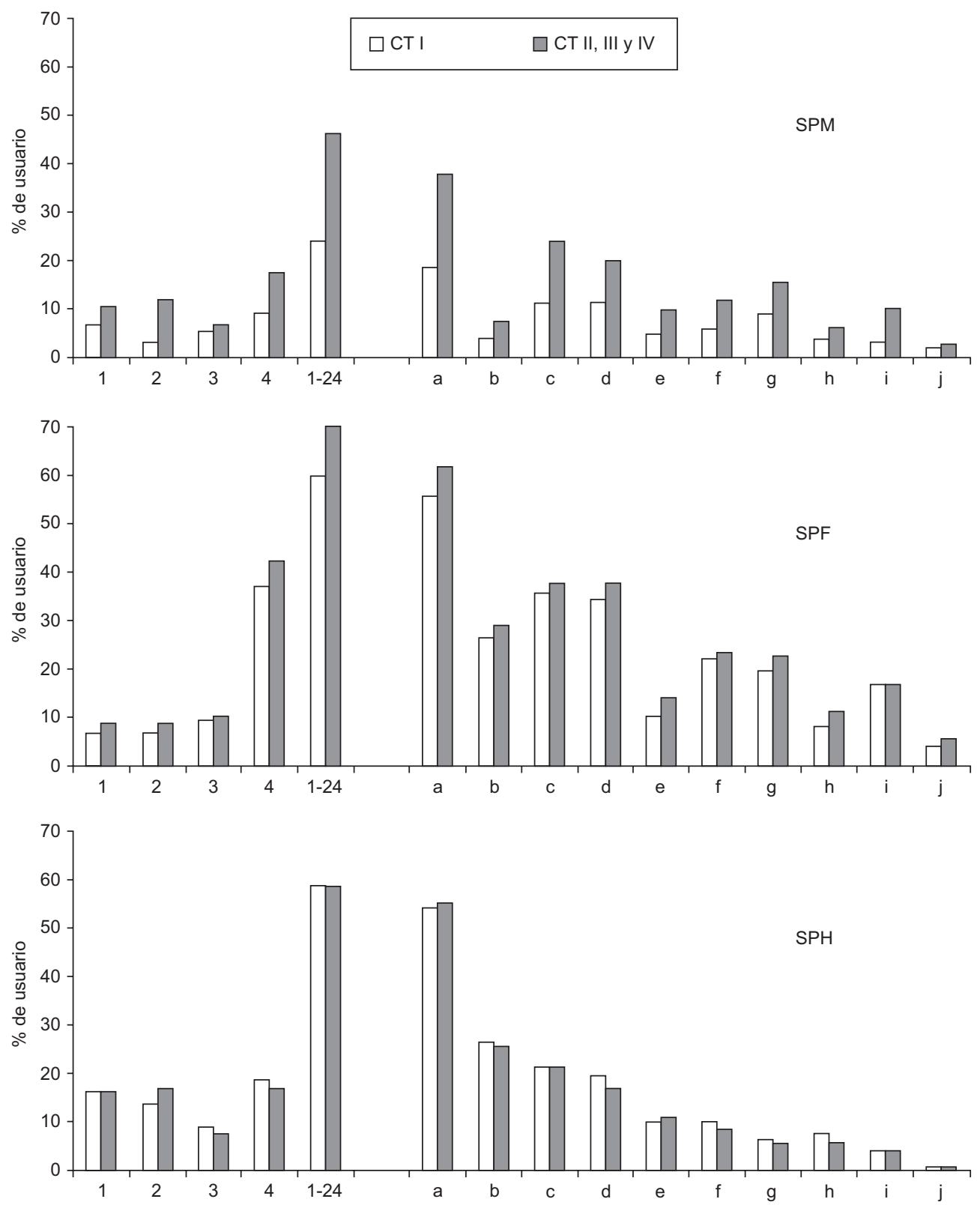

Fig. 2. Síntomas de intoxicación aguda por plaguicidas manifestados por los usuarios en el estudio. SPM: sistema de producción de maíz, $\mathrm{n}=258$; $\mathrm{SPF}$ : sistema de producción de flores, $\mathrm{n}=149$; $\mathrm{SPH}$; sistema de producción de hortalizas, $\mathrm{n}=116$. Categoría toxicológica $(\mathrm{CT})$ : I: extremadamente peligroso; II: altamente peligroso; III: moderadamente peligroso; IV: ligeramente peligroso. 1: $\%$ de usuarios con un síntoma; 2: \% de usuarios con dos síntomas; 3: \% de usuarios con tres síntomas; 4: \% de usuarios con cuatro y más síntomas; 1-24: \% de usuarios con 1 a 24 síntomas. a: dolor de cabeza; b: problemas respiratorios (resequedad de la garganta, flemas, dificultad para respirar, dolor en el pecho); c: náusea/mareo/vómito; d: problemas visuales (irritación, ardor, lagrimeo, visión borrosa); e: problemas en la piel (comezón, ardor); f: dolor abdominal; g: Problemas musculo-esqueléticos (fatiga, debilidad, dolor muscular, calambres/temblor/hormigueo en extremidades, nerviosismo); h: Falta de apetito; i: diarrea; j: salivación 
de México (Hernández-Antonio y Hansen 2011). Los plaguicidas utilizados en el SPH son populares en países centroamericanos (Jansen 2002).

Se observó el uso de varios ingredientes activos (IA) que han sido prohibidos, restringidos o eliminados en Estados Unidos, entre los cuales destacan metamidofos y paraquat. Madeley (2003) indica que el paraquat se ha prohibido, se ha cancelado su registro o se ha restringido severamente en más de 80 países, incluyendo algunos latinoamericanos como Ecuador, Colombia y Costa Rica, donde ya se prohíbe su importación y uso (Instituto Laboral Andino 2006). De acuerdo con la Red Internacional de Acción contra Plaguicidas, 32 IA de los 55 identificados en las diversas categorías toxicológicas (en especial aquellos de la CT I) se encuentran en la lista de plaguicidas altamente peligrosos, y 27 IA están prohibidos en otros países debido a su alta toxicidad aguda y ambiental, e incluso por sus posibles efectos crónicos sobre la salud humana (Cuadro IV). Tal es el caso nuevamente del paraquat por su alta toxicidad aguda; la abamectina, el fosfuro de aluminio, el metamidofos y el metomilo por su toxicidad aguda y ambiental, y el glifosato y el mancozeb por su toxicidad a largo plazo, posiblemente carcinogénica. Al respecto, el glifosato ha sido clasificado como probable carcinógeno por la Agencia Internacional de Investigación del Cáncer (IARC) (PAN Internacional 2015a, b).

Lamentablemente, de acuerdo con el presente estudio muchos de estos IA aún se utilizan y solamente algunos (como metamidofos, paraquat, clorotalonil y quintozeno) son considerados de uso restringido en la legislación mexicana mediante la Ley General de Salud y la Comisión Federal para la Protección contra Riesgos Sanitarios (COFEPRIS 2011). Además, los dos primeros se comercializan libremente sin control y están ampliamente difundidos (el paraquat en el SPM y el metamidofos en el SPH). Algo similar ocurre en Perú (Yengle et al. 2005) y Putumayo, Colombia (Salcedo y Melo 2005).

Los escasos conocimientos y las conductas inapropiadas respecto del uso y manejo de plaguicidas se relacionan con la baja o nula escolaridad de los usuarios. Sin embargo, se identificaron individuos que, a pesar tener una escolaridad más alta, presentan conductas inadecuadas. Lo anterior se explica a partir de la ausencia de capacitación por parte de las empresas comercializadoras, el poco interés de las entidades gubernamentales y la falta de percepción de riesgo de los mismos productores, que si bien conocen algunas características de los plaguicidas no logran traducir ese saber en prácticas adecuadas de manejo, como señalan Isin y Yildirim (2007). La nula escolaridad de los usuarios les impide tener acceso a la información de las etiquetas, e incluso aquellos que saben leer y escribir son alfabetos funcionales; es decir, debido a que su lengua materna es el tzotzil o el tzeltal (lenguas mayas) usan el español como lengua alterna, por lo que su lectura en español es deficiente y no comprenden del todo. De acuerdo con Jørs et al. (2006) se puede afirmar que el desconocimiento, poca compresión y creencias erróneas sobre las prácticas de seguridad, higiene y protección personal, son factores que disminuyen la capacidad de los campesinos para protegerse; ello, además del uso arraigado de estos insumos (Souza y Bocero 2008). Así, las conductas inapropiadas que se identificaron en los tres SP condicionan una exposición más directa a los plaguicidas e incrementan el riesgo de intoxicación en el JF y su familia (Montoro et al. 2009).

La mayoría de los usuarios de plaguicidas en las comunidades estudiadas son jóvenes en edad reproductiva, lo cual incrementa su vulnerabilidad a las consecuencias de salud negativas. La situación de las mujeres JF que pertenecen al SPH es aún más preocupante, ya que debido a su condición de género, edad reproductiva y baja escolaridad, es válido pensar que son aún mucho más vulnerables con relación al resto de la población (Zúñiga et al. 2012). En estas mujeres, la responsabilidad de las actividades agrícolas (específicamente la aplicación de plaguicidas), aunada a las labores domésticas de lavado de ropa contaminada, incrementa el riesgo de exposición y daño a la salud (Salcedo y Melo 2005).

Aunque las IAP no fueron valoradas ni diagnosticadas por personal calificado y pruebas de laboratorio, se puede suponer que el incremento de casos de IAP hasta casi $100 \%$ en el SPM, en el momento o después de fumigar las parcelas, puede estar relacionado con los efectos tóxicos de los herbicidas paraquat (CT II), 2,4-D (CT III) y glifosato (CT VI), como señalan Ferrer (2003), Madeley (2003), Salazar y Aldana (2011) y Harikrishan y Usha (2007), lo cual indica su potencial para causar daño a la salud de los usuarios. Además, el glifosato se ha vinculado en diversas investigaciones con posibles efectos citotóxicos y genotóxicos, lo que aumenta su riesgo potencial para la salud pública (Villaamil et al. 2013).

En el SPF y el SPH la proporción de casos de IAP es muy similar entre las diferentes CT. Esto se debe probablemente a que en el SPM se emplean IA más diferenciados (herbicidas) durante la limpia de las parcelas, mientras que en el SPF y SPH se utilizan insecticidas, fungicidas y herbicidas de distintas CT 
de manera individual y/o mezclados en diferentes momentos durante el ciclo agrícola, lo que dificulta la identificación del IA potencialmente asociado a la sintomatología. Lo anterior pone de manifiesto la complejidad de la exposición a plaguicidas en los tres sistemas. El estudio de los efectos de las mezclas de varios IA es un área poco explorada de la toxicología, por lo que es necesario investigar sus posibles efectos sinérgicos y los posibles daños a la salud pública. Sin embargo, se puede sospechar que los síntomas están asociados al uso de organofosforados, carbamatos y ditiocarbamatos de CT I y II, que son los tipos de plaguicidas más ampliamente utilizados en el SPF y SPH y, de acuerdo con Eddleston et al. (2002), son los responsables de la mayor parte de las intoxicaciones agudas y muertes en el sector agrícola. Los resultados son similares a lo reportado para algunas comunidades de Perú (Montoro et al. 2009), Centroamérica (RAPAC 2003) y Nayarit, México (González Arias et al. 2010).

En el presente estudio solamente se consideraron los malestares que presentaba el JF; sin embargo, durante las labores agrícolas se observó la colaboración de niños, adolescentes y mujeres. De acuerdo con Camarena et al. (2012) dichos actores representan un sector de la población altamente vulnerable a los riesgos de salud que conlleva la exposición accidental o laboral a plaguicidas en el campo agrícola. Por otro lado, debido a que varios de los productos identificados pertenecen a los organofosforados, dicha exposición podría representar un grave riesgo para la salud reproductiva de la familia (Pérez et al. 2012). De mantenerse el uso indiscriminado e incontrolado de los plaguicidas identificados, existe el riesgo de que los usuarios y su familia manifiesten efectos crónicos de salud en el futuro. Esto se debe al potencial tóxico agudo/crónico de los productos utilizados, así como a las posibles consecuencias y desequilibrios ambientales, razones por las cuales diversos ingredientes activos ya fueron prohibidos en otros países, mientras que en México se siguen utilizando sin control y con una legislación laxa en la materia.

Chiapas es uno de los estados que tuvo tendencia al alza en casos de IAP en el periodo 2000 a 2012 (tres a seis casos por cada 100 mil habitantes, respectivamente); sin embargo, Gutiérrez (2013) menciona un subregistro de casos en la entidad, lo cual se confirma en el presente estudio, ya que que se detectaron individuos con síntomas asociados a la intoxicación aguda que no han sido atendidos y, por lo tanto, no están considerados en el registro correspondiente del Sistema Único de Información para la Vigilancia Epidemiológica de la Secretaría de Salud. Por otro lado, Gómez-Arroyo et al. (2000) y Martínez-Valenzuela et al. (2009) asociaron varios de los ingredientes activos identificados con daño genotóxico en campesinos de los estados de Morelos y Sinaloa, México. Por lo tanto, se puede suponer que la salud de los usuarios que utilizan estos productos en la región se encuentra en alto riesgo.

Finalmente, destaca la responsabilidad de los gobiernos federal y estatal, cuyos programas de apoyo al campo y atención a la pobreza están influyendo de manera directa e indirecta en la utilización de plaguicidas. Los recursos monetarios provenientes de Procampo, Oportunidades e incluso Amanecer, en ese orden de importancia, se invierten parcial o completamente en la compra de plaguicidas. Incluso, el Programa Maíz Solidario proporciona herbicidas probablemente asociados con los daños a la salud mencionados anteriormente $(2,4-\mathrm{D}$, paraquat y glifosato).

Los resultados del presente estudio brindan información para que los diferentes actores relacionados con el sector agrícola de la región, propongan y prioricen acciones dirigidas a atender la problemática relacionada con el alto consumo de plaguicidas, y promuevan y favorezcan la salud de la población que se dedica a las actividades agrícolas en los sistemas de producción estudiados.

\section{CONCLUSIONES}

La población agrícola que participó en el estudio tiene escasos conocimientos sobre el uso y manejo adecuado de plaguicidas, carencia que está asociada con baja o nula escolaridad (SPH). Sin embargo, indistintamente del nivel escolar, se observaron conductas inapropiadas de manipulación de dichos productos en los tres sistemas estudiados, lo cual provoca que la población usuaria se encuentre altamente expuesta a los riesgos asociados al uso de dichos productos. Los síntomas de IAP manifestados por el grueso de los campesinos de los diferentes sistemas, mayoritariamente jóvenes y en edad reproductiva, alertan sobre el alto potencial tóxico de los diversos productos químicos que se identificaron en el presente estudio, de los cuales varios están restringidos o prohibidos en la legislación mexicana y en otros países, y el riesgo que representa su uso para la salud. Dichos resultados pueden sugerir la aparición a mediano y largo plazo de consecuencias crónicas para la salud y el ambiente debido al uso de agroquímicos. Es necesario que los diversos actores 
relacionados con esta problemática, principalmente los gubernamentales, se involucren en el establecimiento de una regulación más estricta en materia de uso, manejo y comercialización de plaguicidas extremada y altamente tóxicos en el campo agrícola mexicano, así como en la implementación de estrategias integrales dirigidas a la reconversión a sistemas de producción más amigables con el ambiente y la salud de la población en general.

\section{AGRADECIMIENTOS}

Este estudio fue financiado por el Consejo Nacional de Ciencia y Tecnología (CONACyT) a través del proyecto núm. 132979 denominado "Utilización de plaguicidas y percepción de riesgos en comunidades rurales de los Altos de Chiapas, México". El trabajo forma parte de la tesis doctoral del primer autor, realizado en el Programa de Doctorado en Ecología y Desarrollo Sustentable de El Colegio de la Frontera Sur. El primer autor agradece al CONACyT la beca otorgada para la realización de sus estudios doctorales.

\section{REFERENCIAS}

Álvarez-Solís J.D. y Anzuento-Martínez M.J. (2004). Actividad microbiana del suelo bajo diferentes sistemas de producción de maíz en los altos de Chiapas, México. Agrociencia 38 (1), 13-22.

Ancury T.A., Quandt S.A. y Dearry A. (2001). Farmworker pesticide exposure and community-based participatory research: rationale and practical applications. Environ. Health Perspect. 109 (Supl. 3), 429-433.

DOI: 10.1289/ehp.01109s3429

Camarena O.L, von Glascoe C.A., Arellano G.E., Zúñiga V.E. y Martínez V.C. (2012). Agroquímicos y mujeres indígenas jornaleras en Baja California. En: Género, ambiente y contaminación por sustancias químicas (Cedillo L.A. y Cano F.K., Comps.). Instituto de Nacional de Ecología, Secretaría de Medio Ambiente y Recursos Naturales, México, pp. 67-78.

COFEPRIS (2011). Registros de plaguicidas autorizados por categoría toxicológica. Comisión Federal para la Protección contra Riesgos Sanitarios, Secretaria de Salud, México, 119 pp. [en línea]. http://www. cofepris.gob.mx/AZ/Paginas/Plaguicidas $\% 20 \mathrm{y} \% 20$ Fertilizantes/CatalogoPlaguicidas.aspx 5/12/2017.

CONAPO (2014). Dinámica demográfica 1990-2010 y proyecciones de población 2010-2030. Consejo Nacional de Población, México, 43 pp. [en línea].
http://www.conapo.gob.mx/work/models/CONAPO/ Proyecciones/Cuadernos/15_Cuadernillo_Mexico. pdf 9/09/2014.

CONEVAL (2012). Informe de pobreza y evaluación en el estado de Chiapas 2012. Consejo Nacional de Evaluación de la Política de Desarrollo Social, México, 57 pp. [en línea]. http://www.coneval.org. $\mathrm{mx} /$ coordinacion/entidades/Documents/Chiapas/ principal/07informe2012.pdf 26/05/2013.

Eddleston M., Karalliedde L., Buckley N., Fernando R., Hutchinson G., Isbister G., Konradsen F., Murray D., Piola J.C., Senanayake N., Sheriff R., Singh S., Siwach S.B. y Smit L. (2002). Pesticide poisoning in the developing world: A minimum pesticides list. Lancet 360, 1163-1167. DOI: 10.1016/S0140-6736(02)11204-9

Ferrer A. (2003). Intoxicación por plaguicidas. ANALES Sis. San Navarra .26 (Supl. 1), 155-171.

Gobierno del Estado de Chiapas (2006). Plan de Desarrollo del Estado de Chiapas 2007-2012. Gobierno del Estado de Chiapas, Chiapas, México, 372 pp.

Gómez-Arroyo S., Díaz-Sánchez Y., Meneses-Pérez M.A., Villalobos-Pietrini R. y de León-Rodríguez J. (2000). Cytogenetic biomonitoring in a Mexican floriculture worker group exposed to pesticides. Mutat. Res. 466, 117-124. DOI: 10.1016/S1383-5718(99)00231-4

Gómez-Gómez M., Danglot-Banck C. y Vega-Franco L. (2013). Cómo seleccionar una prueba estadística (segunda de dos partes). Rev. Mex. Pediatr. 80 (2), 81-85.

González-Arías C.A., Robledo-Marenco M.L., MedinaDíaz I.M., Velázquez-Fernández J.B., Girón-Pérez M.I., Quintanilla-Vega B., Ostrosky-Wegman P., PérezHerrera N.E. y Rojas-García A.E. (2010). Patrón de uso y venta de plaguicidas en Nayarit, México. Rev. Int. Contam. Ambient. 26 (3), 221-228.

Guillén P.L., Sánchez Q.C. y Mercado D.S. (2004). Un aporte al estudio psicosocial del uso de tecnología para el control de malezas en cultivos de maíz. Bioagro 16 (001), 31-38.

Gutiérrez S.J.J. (2013). Panorama histórico de morbilidad y mortalidad por intoxicación por plaguicidas en México 1995-2012. Boletín Epidemiológico 33-35 (30), 1-35 (Secretaría de Salud, México).

Harikrishan V.R. y Usha S. (2007). Revisión crítica del 2,4-D. Respuestas a preguntas frecuentes. En: 2,4-D. Razones para su prohibición mundial (Bejarano F., Ed.). Red de Acción de Plaguicidas de América Latina, Red de Acción sobre Plaguicidas y sus Alternativas en México, International POPs Elimination Network. Edo. de México, México, pp. 19-52.

Hernández-Antonio A. y Hansen A.M. (2011). Uso de plaguicidas en dos zonas agrícolas de México y evaluación de la contaminación de agua y sedimentos. Rev. Int. Contam. Ambie. 27 (2), 115-127. 
Herrera-Portugal C., Ocho-Díaz L.H., Franco-Sánchez G. y Díaz-Barriga F. (2005). Daño a ADN en niños expuestos a DDT en un área palúdica de Chiapas, México. Acta Toxicológica Argentina 13 (1), 12-16.

INE (2004). Sistema de consulta de plaguicidas. Fichas técnicas de plaguicidas incluidos en el catálogo de la Comisión Intersecretarial para el Control del Proceso y Uso de Plaguicidas, Fertilizantes y Sustancias Tóxicas 2004. Instituto Nacional de Ecología, México [en línea]. http://www2.ine.gob.mx/sistemas/plaguicidas/ busquedas.html 8/08/2012

Instituto Laboral Andino (2006). Por la prohibición de la "docena sucia". No a los plaguicidas más nocivos. Documento de trabajo núm. 5. Consejo Consultivo Laboral Andino (CCLA), Instituto Laboral Andino y Comité Sindical Andino de Salud Laboral y Medio Ambiente del CCLA, Lima, Perú, 40 pp.

Isin S. y Yildirim I. (2007). Fruit-growers' perceptions on the harmful effects of pesticides and their reflection on practices: The case of Kemalpasa, Turkey. Crop Prot. 26, 917-922.

DOI: $10.1016 /$ j.cropro.2006.08.006

Jansen K. (2002). Plaguicidas y su regulación en Honduras. Revista Ceiba 43 (2), 273-289.

Jørs E., Morant R.C., Aguilar G.C., Huici O., Lander F., Baelum J. y Konradsen F. (2006). Occupational pesticide intoxications among farmers in Bolivia: A cross-sectional study. Environ. Health 5 (1), 10-19. DOI: 10.1186/1476-069X-5-10

Madeley J. (2003). Paraquat: el controvertido herbicida de Syngenta. Berne Declaration, Swedish Society for Nature Conservation, Pesticide Action Network UK, Pesticide Action Network Asia Pacific, Foro Emaús, Red de Acción en Plaguicidas y sus Alternativas para America Latina, Heredia, Costa Rica, 53 pp.

Martínez-Valenzuela C., Gómez-Arroyo S., VillalobosPietrini R., Waliszewski S., Félix-Gastélum R. y Álvarez-Torres A. (2009). Genotoxic biomonitoring of agricultural workers exposed to pesticides in the north of Sinaloa state, Mexico. Environ. Int. 35 (8), 1155-1159.

DOI: 10.1016/j.envint.2009.07.010

Montoro Y., Moreno R., Gomero L. y Reyes M. (2009). Características del uso de plaguicidas químicos y riesgos para la salud en agricultores de la sierra central del Perú. Rev. Peru. Med. Exp. Salud Pública 26 (4), 466-472.

Nivia E. (2000). Mujeres y plaguicidas. Una mirada a la situación actual, tendencias y riesgos de los plaguicidas. Estudio de caso en Palmira, Colombia. ECOFONDO, Pesticide Action Network International, Red de Acción en Plaguicidas Palmira, Palmira, Colombia, $144 \mathrm{pp}$.
Pérez H.N.E., Alvarado M.J.A., Castillo B.M.T. y González N.R.L. (2012). Efectos reproductivos en agricultores expuestos a plaguicidas en Muna, Yucatán. En: Género, ambiente y contaminación por sustancias químicas (Cedillo L.A. y Cano F.K., Comps.). Secretaría de Medio Ambiente y Recursos Naturales, Instituto de Nacional de Ecología, México, pp. 79-94.

PAN Internacional (2015a). Lista de plaguicidas altamente peligrosos de PAN Internacional. Pesticide Action Network International c/o PAN Germany, Hamburgo, Alemania, 36 pp. [en línea]. http://pan-international. org/pan-international-consolidated-list-of-bannedpesticides/ 23/06/2016.

PAN International (2015b). Consolidated list of banned pesticides. 2a ed. Pesticide Action Network International, Hamburgo, Alemania [en línea]. http:// pan-international.org/pan-international-consolidatedlist-of-banned-pesticides/ 23/06/2016.

Plenge-Tellechea F., Sierra-Fonseca J.A. y Castillo-Sosa Y. A. (2007). Riesgos a la salud humana causados por plaguicidas. Tecnociencia Chihuahua 1 (3), 4-6.

RAPAC (2003). Sin plaguicidas. Tierra sana, vida sana. Alternativas comprobadas para sustituir a los 12 plaguicidas más dañinos en Centroamérica. Red de Acción en Plaguicidas y sus Alternativas en América Central, Comité Nacional de Manejo Integrado de Plagas, Programa del Centro Agronómico Tropical de Investigación y Enseñanza/Manejo Integrado de Plagas y Agroforestería, Managua, Nicaragua, 19 pp.

Ríos-González A. (2006). Efecto de plaguicidas inhibidores de colinesterasa en niños de 8-14 años de la región Frailesca, Chiapas. Tesis de Maestría. El Colegio de la Frontera Sur, Chiapas, México, 29 pp.

SAGARPA (2007). Programa de Apoyos Directos al Campo (Procampo). Antecedentes. Secretaría de Agricultura, Ganadería, Desarrollo Rural, Pesca y Alimentación, México [en línea]. http://www.aserca. gob.mx/artman/publish/article_184.asp 28/04/2014

Salazar L.N.J. y Aldana M.M.L. (2011). Herbicida glifosato: usos, toxicidad y regulación. Biotecnia XIII (2), 23-28.

Salcedo M.A. y Melo T.O.L. (2005). Evaluación del uso de plaguicidas en la actividad agrícola del Departamento de Putumayo. Revista Ciencias de la Salud 3 (2), 168-185.

Santiago-Lastra J.A. y Perales-Rivera H.R. (2007). Producción campesina con alto uso de insumos industriales: el cultivo de repollo (Brassica aleracea var. capitata) en los Altos de Chiapas. Ra Ximhai 3 (2), 481-507.

Schoijet M. (2011). La problemática de los plaguicidas químicos: elementos históricos y actuales. Segunda época 5 (10), 53-68. 
Souza-Casadinho O.J. y Bocero S.L. (2008). Agrotóxicos: condiciones de utilización en la horticultura de la Provincia de Buenos Aires (Argentina). Revista Iberoamericana de Economía Ecológica 9, 87-101.

Tinoco R., Parsonnet J. y Halpering D. (1993). Paraquat poisoning in southern Mexico: A report of 25 cases. Arch. Environ. Health 48 (2), 78-80.

DOI: $10.1080 / 00039896.1993 .9938398$

Tinoco O.R. (2005). La construcción local de padecimiento: intoxicaciones por plaguicidas en localidades tojolabales. En: Actores y realidades en la frontera sur de México (Ángeles H., Huicochea L., Saldívar A. y Tuñón E., Coords.) Consejo Estatal de Población, El Colegio de la Frontera Sur, Chiapas, México, pp. 261- 283.

Triola M. F. (2004). Estadística. 9a ed. Pearson Educación, México, $842 \mathrm{pp}$.

Varona M.E., Tolosa J.E., Cárdenas O., Torres C.H., Pardo D., Carrasquilla G. y Frumkin H. (2005). Descripción del uso y manejo de plaguicidas en las empresas de flores afiliadas a Asocolflores. Revista Biomédica 25 (3), 377-389. DOI: 10.7705/biomedica.v25i3.1362

Ventura M.A. (2007). Género y plaguicidas: conocimiento, exposición e intoxicación de mujeres residentes en áreas rurales de la región frailesca, Chiapas. Tesis de Maestría. El Colegio de la Frontera Sur, Chiapas, México, 96 pp.
Villaamil L.E.C., Bovi M. G. y Nassetta M. (2013). Situación actual de la contaminación por plaguicidas en Argentina. Rev. Int. Contam. Ambie. 29, 25-43.

Villarreal G.M.G., López C.E., Bernal P., Escobedo J. y Valadez L. (2009). Rendimiento académico de alumnos de secundaria beneficiarios del Programa Oportunidades en comunidades rurales y semiurbanas de Chiapas y Nuevo León. Región y Sociedad XXI (45), 127-164.

Yengle M., Palhua R., Lascano P.; Villanueva E., Chachi E., Yana E., Zaravia R., Ambrosio J., Clemente J., Cornejo J. y Gutiérrez C. (2005). Prácticas de utilización de plaguicidas en agricultores en el desierto de Huaral-Perú. Revista Peruana de Epidemiología 12 (1), 1-6.

Zúñiga V.E, Arellano G.E., Camarena O.L, Daesslé H.W., Von-Glascoe C., Leyva J.C. y Ruiz B. (2012). Daño genético y exposición a plaguicidas en trabajadores agrícolas del Valle de San Quintín, Baja California, México. Rev. Salud Ambient. 12 (2), 93-101. 